\title{
Como Medir y Guiar Cambios hacia Entornos Educativos más Motivadores
}

\author{
Mónica Martínez-Gómez ${ }^{* 1}$ y Juan A. Marin-Garcia ${ }^{2}$ \\ Universidad Politécnica de Valencia, (1) Departamento de Estadística, Investigación Operativa \\ Aplicadas y Calidad (DEIOAC), (2) ROGLE- Departamento de Organización de Empresas (DOE), \\ Camino de Vera s/n, Valencia-España (e-mail: momargo@eio.upv.es, jamarin@omp.upv.es) \\ *autor a quien debe ser dirigida la correspondencia
}

\begin{abstract}
Resumen
Se presenta la adaptación de un instrumento, utilizado ampliamente en el mundo empresarial para el rediseño de puestos de trabajo, para ser usado en mejorar e incrementar la participación de los estudiantes en su proceso de aprendizaje. Se ha creado una adaptación del Diagnostico de Puestos de Trabajo, la versión castellana del modelo original conocido como Job Diagnostic Survey, JDS. Este consiste en siete escalas que miden las características del trabajo, más un indicador del perfil motivador del puesto y seis escalas de satisfacción con diversos aspectos del citado puesto. Este instrumento ha sido adaptado para ser usado como herramienta de diagnóstico de metodologías docentes en las aulas universitarias y se comprobó su validez y fiabilidad como instrumento de medida. Por último, se investigó la estructura del JDS mediante un análisis factorial, tanto exploratorio como confirmatorio, evaluando distintas hipótesis sobre la estructura del mismo.
\end{abstract}

Palabras clave: metodologías activas, entornos educativos, participación del estudiante, enseñanza universitaria, análisis factorial confirmatorio

\section{How to Measure and Guide Changes towards more Motivating Learning Environments}

\begin{abstract}
An instrument widely used in by several types of companies has been redesigned to guide the process of increasing the involvement of student in their learning process. An adaptation of the instrument called Job Diagnostic Survey (JDS) has been developed in this work. This instrument consists of seven scales for measuring the job characteristics, plus an indicator of the motivating profile of the job position and six levels of satisfaction that cover various aspects of the corresponding job position. This JDS has been used as a diagnostic tool of the teaching methodologies used in university courses and its validity and reliability as a measuring instrument were verified. Finally, the structure of the JDS was investigated by factorial analysis, both exploratory and confirmatory, evaluating different hypotheses about its structure.
\end{abstract}

Keywords: active methodologies, learning environments, student participation, university teaching, confirmatory factorial analysis 


\section{INTRODUCCIÓN}

El Job Diagnostic Survey (JDS) es uno de los instrumentos de diagnóstico que ha sido utilizado para medir y guiar el proceso de rediseño de puestos de trabajo (Boonzaier et al., 2001; Griffeth, 1985; Terborg y Davis, 1982). Este proceso de rediseño consiste en dotar a los operarios de puestos donde puedan sentirse activos, participar en las decisiones, implicarse en sus resultados y disponer de mayor autonomía.

En los últimos años están apareciendo reflexiones sobre la analogía entre el mundo de la empresa y el mundo académico. Desde diversas ramas de la gestión de empresas y de la ingeniería se está reflexionando sobre la posibilidad de aplicar sus teorías para gestionar un grupo como son las clases universitarias (Armstrong, 2003; Bolton, 1999; Donaldson, 2002; Freed, 2005; O'Neil y Hopkins, 2002; Swenson, 2001). Incluso, desde la propia pedagogía se apunta la posibilidad de entender al profesor como un mando que gestiona a un grupo de personas (French y Chopra, 2006). Esto permite al docente poder emprender acciones de mejora de sus asignaturas usando modelos contrastados en otros campos, como, por ejemplo, la administración de empresas.

Por otra parte, diversos autores han manifestado las ventajas de utilizar metodologías activas con alumnos universitarios (Anson et al., 2003; Christoforou et al., 2003; Dyson, 2008; Fruchter, 2001; Gueldenzoph, 2007; Humphreys et al., 2001; Kalliath y Laiken, 2006; Labrador Piquer y Andreu Andrés, 2008; Sheppard et al., 2004; Smart y Csapo, 2007; Watts et al., 2006; Worley, 2008), es decir, de aumentar el nivel de participación o implicación de los estudiantes en el proceso de aprendizaje (Anson et al., 2003; Sheppard et al., 2004; Van Dijk y Jochems, 2002). Sin embargo, los profesores universitarios, al menos dentro del contexto español, perciben ciertas carencias y desinformación, acerca de las verdaderas ventajas e inconvenientes de este tipo de metodologías, especialmente al compararlas con las metodologías expositivas tradicionales, basadas en lección magistral (Lloret y Marin-Garcia, 2008).

Una manera de lograr las metodologías activas es rediseñando el modo en el que los estudiantes realizan sus tareas en clase. Es decir, rediseñar su puesto de trabajo. Si esto se puede realizar usando como herramienta docente los propios contenidos de la asignatura, el proceso será motivador y formativo para los alumnos (Fornaciari y Dean, 2005).

\section{Análisis y rediseño de puestos de trabajo}

De acuerdo con la terminología de estudio del trabajo, un "puesto de trabajo" consiste en una o más funciones desempeñadas por una persona en una empresa en un momento dado (Cascio, 1989). Se considera que esta definición se puede aplicar al contexto educativo si en lugar de "trabajador contratado" se utiliza el término "alumno matriculado" y en lugar de "empresa" se utiliza "asignatura". En el mundo empresarial, hay un enfoque tradicional para el diseño de puestos de trabajo que se basa en obtener la máxima simplificación y especialización funcional posible (Cascio, 1989). Paralelamente, en el mundo docente universitario, hay un enfoque metodológico tradicional que se caracteriza por que el profesor asume como rol prioritario el suministrar información o contenidos a los alumnos a través de la lección magistral (Anson et al., 2003; Wenger y Hornyak, 1999).

El diseño de trabajo tiene como propósito especificar cómo se debe realizar el trabajo para optimizar la consecución de los objetivos de la empresa y favorecer que los trabajadores puedan sentirse satisfechos realizando su trabajo (Cascio, 1989). En nuestra analogía, el diseño del trabajo tendría que ver con la metodología docente propuesta. Es decir, con el modo en que se trabaja en la asignatura para aprender.

Entre las alternativas propuestas a la docencia tradicional se pueden encontrar las metodologías activas (Anson et al., 2003; Fruchter, 2001) que suelen emplear discusiones guiadas por el profesor, participación de los alumnos planteando preguntas que son respondidas en el aula, trabajo en grupo, o workshops, (Anson et al., 2003). La participación activa del alumno en el 
proceso de aprendizaje, pasando de una recepción de información a la evaluación y organización de los conocimientos (Wenger y Hornyak, 1999), proporciona una retención de los conocimientos más duradera (Fornaciari y Dean, 2005).

El objetivo de esta investigación es presentar la adaptación realizada al JDS para usarlo como herramienta de diagnóstico de metodologías docentes en las aulas universitarias y comprobar su validez y fiabilidad como instrumento de medida. Por último, se investigará la estructura factorial del JDS mediante un análisis factorial, tanto exploratorio como confirmatorio, evaluando distintas hipótesis sobre las estructura del mismo.

\section{Propiedades Psicométricas del JDS}

EI JDS (Hackman et al., 1978; Hackman y Oldham, 1980; Hackman y Oldham, 1975) es una de las principales herramientas para evaluar el potencial motivador de los puestos de trabajo (Fried, 1991; Griffin, 1991) y ha sido ampliamente utilizado en el mundo empresarial.

En este trabajo se ha optado por la versión original por diversos motivos. En primer lugar, las únicas adaptaciones al castellano que conocemos con validación empírica del modelo (Fuertes Martínez et al., 1996; González, 1997) utilizan la versión original y hemos preferido mantener la concordancia con esos modelos para poder comparar nuestros índices psicométricos con los obtenidos por esos autores. También resulta muy útil la información que podamos extraer sobre la satisfacción extrínseca al ser variables que pueden modificar los propios profesores. Además, los datos utilizados forman parte de un experimento longitudinal y no queremos cambiar el instrumento de medida para poder hacer comparaciones fiables de la evolución de una asignatura a lo largo de 4 cursos. No obstante, se han eliminado las variables relacionadas con los estados psicológicos críticos como recomiendan la mayoría de los autores (Boonzaier et al., 2001; Taber y Taylor, 1990). Además, se ha elegido el modelo aditivo del potencial motivador (Boonzaier et al., 2001; Tepper et al., 1996) en lugar del modelo multiplicativo original.

Las propiedades psicométricas del modelo JDS son aceptables. La fiabilidad de las 5 escalas originales de características del trabajo se sitúa en torno a 0,70 y la escala de satisfacción general está por encima del 0,85 (Fried, 1991; Morgeson y Humphrey, 2006). Sin embargo, se encuentran estudios donde se proponen soluciones de 2, 3 y 4 factores en lugar del modelo original de 5 factores ortogonales (Rungtusanatham y Anderson, 1996; Taber et al., 1985). Las investigaciones precedentes han utilizado los siguientes procedimientos para probar las propiedades psicométricas del JDS: a) la consistencia interna de las escalas y estructura factorial; b) que la escala sea capaz de detectar diferencias entre puestos diferentes; c) correlaciones externas; d) coherencia test-retest; e) diferencias de las puntuaciones del JDS en puestos similares y f) comparación con métodos alternativos para medir el mismo fenómeno (Morgeson y Humphrey, 2006; Taber y Taylor, 1990).

Este trabajo se ha centrado sólo en los 3 primeros procedimientos, y a su vez, el análisis de la estructura factorial del JDS se centrará únicamente en los ítems que corresponden a los puestos de trabajo, siguiendo la tradición de autores como Taber y Taylor (1990) y Gónzález (1997). En estudios posteriores se completará el estudio de la estructura factorial tanto con los ítems relativos a las características del puesto de trabajo, como a los ítems relativos a la satisfacción del puesto de trabajo.

\section{METODOLOGÍA}

Se ha creado una adaptación de la versión castellana (Fuertes Martínez et al., 1996; González, 1997) del modelo original (Hackman y Oldham, 1980) que sigue siendo considerado como válido (Boonzaier et al., 2001; Griffin, 1991; Taber et al., 1985). El modelo, que se muestra en la tabla 1, consiste en siete escalas que miden las características del trabajo más un indicador del perfil motivador del puesto (PMP) y seis escalas de satisfacción con diversos aspectos del citado puesto. 
Las escalas de las características del puesto de trabajo están medidas con tres ítems valorados de 1 a 7 . En cada escala, uno de los ítems se presenta con frases de anclaje. Los otros dos son una frase a la que se tiene que responder el grado de acuerdo o desacuerdo. Una de esas frases está redactada positivamente y la otra negativamente.

Tabla 1: Definición de las variables del modelo adaptado

\begin{tabular}{|c|c|c|c|}
\hline Abreviatura & $\begin{array}{l}\text { características } \\
\text { del trabajo }\end{array}$ & $\begin{array}{l}\text { Definición adaptada al entorno } \\
\text { educativo }\end{array}$ & Calculo \\
\hline VAR & $\begin{array}{l}\text { Variedad de } \\
\text { habilidades }\end{array}$ & $\begin{array}{l}\text { Número y diversidad de habilidades } \\
\text { para llevar a cabo las actividades }\end{array}$ & {$[(1.4)+(2.1)+(8-(2.5))] / 3$} \\
\hline IDENT & $\begin{array}{l}\text { Identidad de la } \\
\text { tarea }\end{array}$ & $\begin{array}{l}\text { Medida en que la asignatura implica } \\
\text { terminar una unidad completa de } \\
\text { trabajo, identificable en el producto } \\
\text { final }\end{array}$ & $\begin{array}{l}{[(1.3)+(2.11)+(8-(2.3))] /} \\
3\end{array}$ \\
\hline SIGN & $\begin{array}{l}\text { Significación de } \\
\text { la tarea }\end{array}$ & $\begin{array}{l}\text { Impacto que las actividades tienen } \\
\text { sobre otras personas o utilidad para } \\
\text { el futuro profesional }\end{array}$ & {$[(1.5)+(2.8)+(8-(2.14))] / 3$} \\
\hline AUTONOM & Autonomía & $\begin{array}{l}\text { Grado de independencia y } \\
\text { discreción que el estudiante disfruta } \\
\text { para planificar y llevar a cabo su } \\
\text { trabajo }\end{array}$ & {$[(1.2)+(2.13)+(8-(2.9))] / 3$} \\
\hline $\mathrm{Rp}$ & $\begin{array}{l}\text { Retroinformación } \\
\text { del puesto }\end{array}$ & $\begin{array}{l}\text { Grado de información directa que } \\
\text { reciben los estudiantes sobre } \\
\text { resultados y rendimientos de su } \\
\text { trabajo }\end{array}$ & {$[(1.7)+(2.4)+(8-(2.12))] / 3$} \\
\hline Rs & $\begin{array}{l}\text { Retroinformación } \\
\text { social }\end{array}$ & $\begin{array}{l}\text { Grado en que el estudiante recibe } \\
\text { información clara sobre la eficacia } \\
\text { de su trabajo, por los supervisores o } \\
\text { compañeros de trabajo }\end{array}$ & {$[(1.6)+(2.10)+(8-(2.7))] / 3$} \\
\hline CONTACT & Contacto social & $\begin{array}{l}\text { Grado de interacción con otras } \\
\text { personas para llevar a cabo las } \\
\text { actividades }\end{array}$ & {$[(1.1)+(2.2)+(8-(2.6))] / 3$} \\
\hline PMP & $\begin{array}{l}\text { Potencial } \\
\text { Motivador de la } \\
\text { asignatura }\end{array}$ & $\begin{array}{l}\text { Resumen en un solo indicador de las } \\
\text { características de trabajo anteriores. } \\
\text { Usaremos la versión aditiva para el } \\
\text { cálculo }\end{array}$ & $\begin{array}{l}\text { (VAR+IDNT+SIGN+AUTONOM } \\
+\mathrm{RP}) / 5\end{array}$ \\
\hline \multicolumn{4}{|c|}{ Satisfacción } \\
\hline SATg & $\begin{array}{l}\text { Satisfacción } \\
\text { general }\end{array}$ & $\begin{array}{l}\text { Medida global del grado en que el } \\
\text { empleado se siente satisfecho y a } \\
\text { gusto con el puesto }\end{array}$ & $\begin{array}{l}{[(3.3)+(3.13)+(8-(3.9))+} \\
(5.2)+(8-(5.8))] / 5\end{array}$ \\
\hline SATnota & $\begin{array}{l}\text { Satisfacción con } \\
\text { salario }\end{array}$ & Satisfacción con la nota recibida & {$[(4.2)+(4.9)] / 2$} \\
\hline SATsegu & $\begin{array}{l}\text { Satisfacción con } \\
\text { seguridad }\end{array}$ & Seguridad de aprobar & {$[(4.1)+(4.11)] / 2$} \\
\hline SATsoc & $\begin{array}{l}\text { satisfacción } \\
\text { social }\end{array}$ & Satisfacción con los compañeros & {$[(4.4)+(4.7)+((4.12)] / 3$} \\
\hline SATsuper & $\begin{array}{l}\text { Satisfacción con } \\
\text { supervisión }\end{array}$ & Satisfacción con el profesor & {$[(4.5)+(4.8)+(4.14)] / 3$} \\
\hline SATauto & $\begin{array}{l}\text { Satisfacción con } \\
\text { autorrealización }\end{array}$ & $\begin{array}{l}\text { Oportunidades de crecimiento y } \\
\text { desarrollo en la asignatura }\end{array}$ & $\begin{array}{l}{[(4.3)+(4.6)+(4.10)+(4.13)]} \\
/ 4\end{array}$ \\
\hline
\end{tabular}

Debido a las limitaciones de espacio establecidas en las normas de la presente publicación, no se ha podido incluir la definición de los ítems en el artículo. Sin embargo quedan a disposición de cualquier persona interesada, pudiéndolos solicitar al autor de contacto.

Para la construcción de los ítems adaptados al entorno docente, se ha procedido de la siguiente manera: un profesor y 2 alumnos de último año de carrera de ingeniería industrial realizaron, de manera independiente, una conversión de los ítems de la versión castellana del JDS (Fuertes Martínez et al., 1996; González, 1997) a lo que consideraban equivalente en el contexto de 
actividades de alumnos universitarios. Posteriormente, para cada uno de los ítems del modelo, se compararon las tres propuestas, eligiéndose la formulación que resultaba más intuitiva para los estudiantes y verificándose que el ítem resultante concordaba con el significado que tenía el ítem equivalente del modelo original.

Para evaluar si las escalas de medida siguen siendo adecuadas, se procedió a evaluar la fiabilidad y validez de las mismas. La fiabilidad se define como el grado de consistencia entre múltiples medidas de una variable y se estima midiendo la consistencia interna de una variable. La consistencia interna se refiere a que los ítems individuales de la escala deberían estar midiendo las mismas cosas y por lo tanto estar altamente correlacionados. Para la fiabilidad de las escalas se utilizó como medida de bondad el a de cronbach (Fuertes Martínez et al., 1996; González, 1997; Taber y Taylor, 1990), aunque esta medida de fiabilidad presenta el inconveniente de asumir que existe unidimensionalidad de cada constructo en lugar de suponerlo (Hair et al., 1995). Esta medida nos permite calcular la fiabilidad de la escala de manera conjunta y es muy sensible al número de indicadores que definen cada constructo. Otras medidas utilizadas han sido la fiabilidad compuesta y la varianza extraída siendo valores apropiados para cada caso los que exceden a 0,7 y 0,5 respectivamente (Hair et al., 1995).

Finalmente, se utilizará la alternativa del análisis factorial confirmatorio, para analizar la fiabilidad de la escala y verificar la estructura subyacente del cuestionario. Se formularon distintas hipótesis o modelos, frente al modelo originalmente propuesto de 5 factores ortogonales (González, 1997). Se hipotetizaron modelos con otras estructuras factoriales como las propuestas por este mismo autor (González, 1997): modelo de un factor (modelo 1a), modelo de tres factores, identidad, feedbaack y otros tanto sin ítems negativos como con ellos, (modelos 2a y 2b), modelo con 5 factores, tanto ortogonales como correlacionados y tanto considerando todos los ítems como sin considerar los formulados en sentido inverso (modelos 3a, 3b, 3c, 3d, 3e y 3f) y otro de 7 factores tanto ortogonales como correlacionados y tanto considerando todos los ítems como sin considerar los formulados en sentido inverso (modelos $4 a, 4 b, 4 c$ y $4 d$ ).

El proceso de estimación utilizado fue el de Máxima Verosimilitud (ML) bajo la hipótesis de normalidad multivariante, por ser el procedimiento más recomendado cuando existen como en nuestro caso, datos faltantes. La estimación de los distintos modelos hipotetizados se llevó a cabo mediante el programa EQS 6.1.

Para evaluar la bondad de ajuste de los distintos modelos propuestos se evaluaron todos los índices respecto de sus valores de corte habituales (Bentler, 1990; Bentler y Bonnet, 1980; Bollen y Long, 1993; Bollen, 1989; Brown y Cudek, 1993; Jöreskog, 1993; Jöreskog y Sörborn 1981; Jöreskog y Sörbom 1986; Mulaik et al. (1989), siendo necesario evaluar el máximo número de ellos, especialmente cuando se comparan modelos entre sí (Mulaik et al., 1989).

En este caso, las variables de medida son los ítems del cuestionario, correlacionados en los distintos modelos con las variables latentes correspondientes. Se procuró que todas las variables manifiestas necesarias estuvieran incluidas en cada uno de los modelos, de manera que se evitara cometer errores de especificación.

Los datos se recogieron en el curso 2005-06. El cuestionario adaptado se administró a 103 estudiantes de $4^{\circ}$ curso de la titulación de ingeniería de organización. Cada uno de los alumnos rellenaba dos cuestionarios, uno para la asignatura con metodologías activas (EPOLIE) y otro para una de las asignaturas con metodología tradicional.

\section{RESULTADOS Y DISCUSIÓN}

La tabla 2 resume los análisis realizados que nos permitirán comprobar la fiabilidad de las escalas adaptadas y las compara con los datos del modelo original referente a puestos de trabajo. En ella se puede apreciar que las propiedades psicométricas del modelo adaptado son discretas. Tres de las características de trabajo (variedad, identidad y autonomía) y una de las satisfacciones 
(satisfacción con la nota), tienen un a de Cronbach muy bajo e inferior a los índices obtenidos en las investigaciones realizadas con el cuestionario original para empresas (Taber y Taylor, 1990).

Tabla 2: Índice de consistencia interna de las escalas calculada con Omega de Heiser

\begin{tabular}{lccccc}
\hline \multicolumn{1}{c}{ Escala } & Noitems & Docente & $\begin{array}{c}\text { (Fuertes Martínez et } \\
\text { al., 1996) }\end{array}$ & (González, 1997) & $\begin{array}{c}\text { (Taber y Taylor, } \\
\text { 1990) }\end{array}$ \\
\hline VAR & 3 & 0,46 & 0,22 & 0,8 & 0,70 \\
IDENT & 3 & 0,46 & 0,30 & 0,78 & 0,67 \\
SIGN & 3 & 0,76 & 0,44 & 0,71 & 0,65 \\
AUTONOM & 3 & 0,40 & 0,31 & 0,73 & 0,69 \\
Rp & 3 & 0,79 & 0,46 & 0,70 & 0,70 \\
Rs & 3 & 0,79 & 0,49 & n.d & n.d \\
CONTACT & 3 & 0,74 & 0,38 & n.d & n.d \\
PMP & 15 & 0,75 & 0,50 & n.d & n.d \\
SATg & 5 & 0,82 & 0,76 & n.d & n.d \\
SATnota & 2 & 0,36 & 0,79 & n.d & n.d \\
SATsegu & 2 & 0,64 & 0,62 & n.d & n.d \\
SATsoc & 3 & 0,80 & 0,45 & n.d & n.d \\
SATsuper & 3 & 0,76 & 0,68 & n.d & n.d \\
SATauto & 4 & 0,78 & 0,77 & n.d & n.d \\
Número de & & 206 & 133 & 230 & Meta-análisis \\
casos & & & & & \\
\hline
\end{tabular}

También son menores a los obtenidos por González (1997) en su adaptación del cuestionario al castellano, aunque este autor ya advierte que el índice que utiliza es diferente del $\alpha$ de Cronbach, que sería menor que los datos presentados. Sin embargo, los valores de las escalas del cuestionario docente son, en general, bastante superiores a los que se alcanzaron con la versión castellana del JDS para puestos de trabajo (Fuertes Martínez et al., 1996). Respecto a las escalas de satisfacción, excepto la de satisfacción con la nota, todas muestran unos valores de consistencia interna adecuados.

En la tabla 3 se presentan las correlaciones unilaterales entre las dimensiones del JDS y la satisfacción. En general, los datos de nuestra investigación concuerdan con investigaciones anteriores (Fried, 1991) y se reproducen las correlaciones moderadas y significativas entre la satisfacción general, en torno al 0,5 , con casi todas las dimensiones del JDS.

Tabla 3: Correlaciones unilaterales entre las dimensiones del JDS y la satisfacción de los estudiantes. Correlación de Pearson significativo al: ${ }^{*} \alpha<5 \%{ }^{* *} \alpha<1 \%$.

\begin{tabular}{|c|c|c|c|c|c|c|}
\hline & SATg & SATnota & SATSegu & SATsoc & SatSuper & SATautorre \\
\hline VAR &, $414\left({ }^{* \star}\right)$ &, $138\left(^{*}\right)$ &,$- 145\left(^{*}\right)$ &, $586\left({ }^{* *}\right)$ &, $461\left(^{* *}\right)$ &, $501\left(^{* *}\right)$ \\
\hline IDENT &, $375\left({ }^{* *}\right)$ &, $\left.1811^{* *}\right)$ &, $\left.183^{(* *}\right)$ &, $306\left({ }^{* *}\right)$ &, $396\left(^{* *}\right)$ &, $429\left(^{* *}\right)$ \\
\hline SIGN &, $506\left({ }^{* *}\right)$ &, $135\left(^{*}\right)$ & ,003 &, $689\left(^{* *}\right)$ &, $528\left(^{* *}\right)$ &, $580\left(^{* *}\right)$ \\
\hline AUTONOM &, $383\left(^{* \star}\right)$ & ,081 &, $126\left(^{*}\right)$ &, $345\left(^{* *}\right)$ &, $\left.240{ }^{* *}\right)$ &, $401\left({ }^{* *}\right)$ \\
\hline $\mathrm{Rp}$ &, $525\left({ }^{* *}\right)$ &, $225\left(^{* *}\right)$ &, $247\left(^{* *}\right)$ &, $512\left({ }^{* *}\right)$ &, $565\left(^{* *}\right)$ &, $595\left(^{* *}\right)$ \\
\hline Rs &, $512\left({ }^{\star \star}\right)$ &, $115\left(^{*}\right)$ & ,024 &, $682\left({ }^{* \star}\right)$ &, $\left.6511^{* *}\right)$ &, $669\left(^{* \star}\right)$ \\
\hline CONTACT &, $440\left(^{* \star}\right)$ & ,087 &,$- 162\left(^{*}\right)$ &, $644\left({ }^{* \star}\right)$ &, $464\left(^{(* *}\right)$ &, $558\left(^{\star \star}\right)$ \\
\hline PMP &, $612\left({ }^{* *}\right)$ &, $181\left(^{* *}\right)$ & ,039 &, $747\left({ }^{* *}\right)$ &, $\left.650^{(* *}\right)$ &, $729\left(^{* *}\right)$ \\
\hline
\end{tabular}

En la tabla 4, se muestran los valores obtenidos para la fiabilidad del constructo y la varianza extraída para los modelos de 5 y 7 factores, tanto considerando los ítems formulados en sentido negativo como los que no los consideraban. Los únicos constructos con la fiabilidad y varianza extraída con valores adecuados son la significación y la retroinformación del puesto en los casos de los modelos de 5 factores, mientras que en el caso de siete factores, lo son la significación, la 
retroinformación del puesto, la retroinformación social y el contacto social, coincidiendo con lo anteriormente comentado de que la validez de alguna de las escalas no esta establecida con contundencia.

Tabla 4: Fiabilidad de constructo (FC) y varianza extraída (VE). Valores adecuados superiores o iguales a 0,7 y 0,5 respectivamente.

\begin{tabular}{lcccccccc}
\hline & \multicolumn{2}{c}{5 Factores } & \multicolumn{2}{c}{$\begin{array}{c}5 \text { Factores sin items } \\
\text { negativos }\end{array}$} & \multicolumn{2}{c}{7 Factores } & \multicolumn{2}{c}{7 Factores sin } \\
& \multicolumn{2}{c}{} & \multicolumn{3}{c}{ items negativos } \\
\cline { 2 - 9 } & FC & VE & FC & VE & FC & VE & FC & VE \\
\hline IDENTIDAD (F3) & 0,453 & 0,225 & 0,591 & 0,427 & 0,470 & 0,278 & 0,570 & 0,403 \\
VARIEDAD (F1) & 0,561 & 0,356 & 0,420 & 0,339 & 0,523 & 0,36 & 0,568 & 0,510 \\
SIGNIFICACIÓN (F4) & 0,787 & 0,565 & 0,846 & 0,734 & 0,783 & 0,562 & 0,847 & 0,735 \\
AUTONOMÍA (F2) & 0,462 & 0,249 & 0,439 & 0,352 & 0,544 & 0, & 0,383 & 0,237 \\
& & & & & & 375 & & \\
Rp (F5) & 0,746 & 0,507 & 0,757 & 0,611 & 0,662 & 0,423 & 0,729 & 0,582 \\
Rs (F6) & ------ & ------- & ------- & ----- & 0,737 & 0,488 & 0,787 & 0,665 \\
CONTACT (F7) & ----- & ----- & ---- & -- & 0,802 & 0,613 & 0,932 & 0,873 \\
\hline
\end{tabular}

Los índices de bondad de ajuste de los distintos modelos hipotetizados de muestran en la tabla 5. En todos los modelos hipotetizados, resultaron los de mejores índices de bondad de ajuste los que no presentaban ítems formulados en orden inverso.

Tabla 5: Índices de bondad de ajuste de los modelos hipotetizados

\begin{tabular}{ccccccccccc}
\hline Modelos & $\mathrm{X}^{2}$ & gdl & $\begin{array}{c}\mathrm{p}- \\
\text { values }\end{array}$ & NFI & CFI & PNFI & PCFI & RMSEA & ECVI & HOELTE \\
\hline 1.a & 1023,15 & 74 & 0,000 & 0,00 & 0,00 & 0,00 & 0,00 & 0,207 & 5,586 & 27 \\
2.a & 524,937 & 158 & 0,000 & 0,85 & 0,84 & 0,63 & 0,68 & 0,094 & 3,478 & 86 \\
2.b & 187,31 & 66 & 0,000 & 0,90 & 0,93 & 0,63 & 0,66 & 0,086 & 1,558 & 105 \\
3.a & 162,93 & 75 & 0,000 & 0,81 & 0,87 & 0,87 & 0,89 & 0,089 & 12,229 & 22 \\
3.b & 144,09 & 65 & 0,000 & 0,85 & 0,91 & 0,56 & 0,60 & 0,073 & 1,230 & 128 \\
3.c & 675.40 & 45 & 0,039 & 0,96 & 0,98 & 0,54 & 0,58 & 0,058 & 1,565 & 105 \\
3.d & 24,23 & 25 & 0,2843 & 0,96 & 0,99 & 0,45 & 0,47 & 0,026 & 0,569 & 279 \\
3.e & 28,6 & 26 & 0,330 & 0,96 & 0,99 & 0,45 & 0,47 & 0,022 & 0,569 & 279 \\
3.f & 178,11 & 76 & 0,000 & 0,83 & 0,89 & 0,63 & 0,56 & 0,081 & 1,493 & 113 \\
4.a & 918,45 & 210 & 0,000 & 0,57 & 0,62 & 0,60 & 0,65 & 0,150 & 3,111 & 102 \\
4.b & 401,75 & 133 & 0,000 & 0,83 & 0,90 & 0,60 & 0,65 & 0,082 & 3,260 & 109 \\
4.c & 588,84 & 71 & 0,000 & 0,68 & 0,62 & 0,52 & 0,54 & 0,189 & 1,360 & 178 \\
4.d & 88,899 & 30 & 0,006 & 0,9 & 0,98 & 0,53 & 0,54 & 0,0051 & 1,370 & 178 \\
\hline
\end{tabular}

Analizando los distintos índices de bondad de ajuste, se puede concluir que las mejores representaciones de la estructura factorial del JDS son de naturaleza multidimensional. Examinando los modelos que representan una solución oblicua con tres factores (modelos 2a y $2 b$ ), el ajuste de los datos es satisfactorio, aunque el $\chi^{2}$ continúa denotando divergencias entre la matriz de varianzas y covarianzas de la muestra y la matriz generada a partir del modelo $\left[\chi^{2}\right.$ $=524,937$, gdl=158 y $p$-value $=0,000$ en el primer caso y $\chi^{2}=187,31$, gdl=66 y $p$-value $=0,000$, en el segundo]. El modelo de 5 factores ortogonales (3a), presenta de nuevo ajuste bastante inadecuado, denotando divergencias entre la matriz de varianzas y covarianzas de la muestra y la matriz generada a partir del modelo. Aunque tampoco se reducen dichas divergencias cuando hablamos de una solución oblicua de 5 factores considerando todos los ítems $\left[\chi^{2}=144,099\right.$, gdl=182 y p-value=0,000], los índices de bondad de ajuste mejoran bastante, estando próximos a los valores recomendados para cada uno de ellos. Por otro lado, el la estructura de 5 factores oblícuos sin ítems negativos desarrollada en el modelo $3 \mathrm{c}$, presenta un ajuste moderado, $\left[\chi^{2}\right.$ $=24,2344 p$-value $=0,284]$, y el resto de índices están dentro de los límites recomendados para un buen ajuste: NFI y CFI están próximos a 0,9 (0,958 y 0,994) aunque el modelo no denote mucha parsimonia, pues los índices PNFI y PGFI empeoran, no estando muy próximos a 0,6 (0,453 y $0,471)$, que son los que deben considerarse al comparar modelos alternativos una vez verificados 
los índice de bondad de ajuste de los correspondientes modelos (Mulaik et al., 1989). Sin embargo, el estadístico $\chi^{2} / \mathrm{gdl}$, evidencia un buen ajuste en ambos modelos considerando las posiciones más estrictas de la interpretación. También el error cuadrático medio de aproximación está bastante próximo a cero, lo que denota que la discrepancia entre matriz de covarianzas esperada y observada es mínima. Hoelter toma valores superiores a 200, concretamente 279, indicando que las diferencias entre el modelo planteado y los datos no son relevantes. Finalmente, el índice ECVI debe tomar valores próximos a cero. De nuevo tampoco se reducen las divergencias entre la matriz de varianzas y covarianzas de la muestra y la generada a partir del modelo cuando hablamos de una solución oblicua de 5 factores y un constructo denominado "Método" (modelo 3d) definido a partir de los ítems formulados en sentido inverso $\left[\chi^{2}=178,118, p-\right.$ value $=0,000]$, pero también los índices de bondad de ajuste son aceptables. Los dos modelos de 7 factores correlacionados propuestos ( $4 a$ y $4 b$ ) con todos los ítems y sin considerar los ítems formulados en sentido negativo (4c y $4 \mathrm{~d}$ ), continúan denotando divergencias entre la matriz de varianzas y covarianzas de la muestra y la generada a partir del modelo, aunque mejoran los índices de bondad de ajuste en el caso de no considerar los ítems formulados en sentido negativo], llegando a alcanzar valores muy próximos a los recomendados para cada uno de ellos, especialmente RMSEA, cuyo valor de 0,0051 está muy próximo a cero y HOELTE, con una valor de 178.

De acuerdo con los resultados del análisis de los índices de bondad de ajuste, el modelo hipotetizado con una estructura de cinco factores oblicuos sin ítems en sentido inverso es el que mejor representa la estructura factorial de la versión adaptada del JDS. En la tabla 6 se muestran los parámetros estandarizados para y las correlaciones los factores del mismo.

Tabla 6: Valores estandarizados y correlaciones para el modelo con 5 factores correlacionados sin ítems en sentido inverso (3e)

\begin{tabular}{|c|c|c|c|c|}
\hline Relación & & $\begin{array}{c}\text { Valor } \\
\text { estandarizado } \\
\text { molelo } 3 e\end{array}$ & Correlación & Valor \\
\hline PMP & $<---\quad F 1$ & ,781 & $\mathrm{F} 2 \leftrightarrow \mathrm{F} 1$ & ,680 \\
\hline PMP & $<---\quad$ F2 & ,054 & $\mathrm{F} 1 \leftrightarrow \mathrm{F} 3$ & ,711 \\
\hline PMP & $<---\quad$ F3 &, 067 & $\mathrm{~F} 1 \leftrightarrow \mathrm{F} 4$ & ,851 \\
\hline PMP & $<---\quad F 4$ &, 079 & $\mathrm{~F} 5 \leftrightarrow \mathrm{F} 1$ &, 761 \\
\hline PMP & $<---\quad$ F5 & ,081 & $\mathrm{F} 2 \leftrightarrow \mathrm{F} 3$ & ,654 \\
\hline S1.P4 & $<---\quad F 1$ & ,864 & $\mathrm{F} 2 \leftrightarrow \mathrm{F} 4$ &, 531 \\
\hline S1.P2 & $<---\quad F 2$ & ,600 & $\mathrm{F} 5 \leftrightarrow \mathrm{F} 2$ &, 650 \\
\hline S1.P3 & $<---\quad$ F3 &, 742 & $\mathrm{~F} 3 \leftrightarrow \mathrm{F} 4$ &, 516 \\
\hline S2.P11 & <--- $\quad$ F3 &, 540 & $\mathrm{~F} 5 \leftrightarrow \mathrm{F} 3$ &, 706 \\
\hline S1.P5 & $<---\quad F 4$ & ,870 & $\mathrm{F} 5 \leftrightarrow \mathrm{F} 4$ & 670 \\
\hline S2.P8 & <--- $\quad F 4$ & ,836 & & \\
\hline S1.P7 & $<---\quad$ F5 & ,890 & & \\
\hline S2.P4 & $<---\quad$ F5 & ,682 & & \\
\hline S2.P5 & <--- $\quad$ F1 &, 539 & & \\
\hline S2.P9 & $<---\quad$ F2 & ,443 & & \\
\hline S2.P14 & $<---\quad F 4$ & ,485 & & \\
\hline S2.P12 & <--- $\quad$ F5 &, 509 & & \\
\hline
\end{tabular}

En consecuencia, existe bastante variabilidad en el número de factores con los que se puede modelizar la estructura del JDS adaptado a metodologías docentes, tal y como sucedía con la versión del JDS original (Idaszak et al., 1988). El principal inconveniente ya detectado por estos autores y, originalmente propuesto por Lawley y Maxwell (1971), es que cada factor únicamente viene representado por tres ítems y ello causa serios problemas. Finalmente establecer, que los modelos que mejor ajuste presentan son los que no consideran los ítems formulados en sentido inverso, ya que dichos ítems pueden conducir a otros problemas cuando se trata de factores con muy pocos ítems, como es nuestro caso (Idaszak et al., 1988). Además, Dunham et al. (1977), establecen que la estructura original propuesta de 5 factores no puede ser considerada única para todas las muestras, y dependerá de la muestra que esté siendo utilizada en cada caso. 


\section{CONCLUSIONES}

La confirmación de 7 factores que sustentan el perfil motivador de una asignatura permite abordar la mejora docente desde un enfoque analítico, creando 7 vías de acción. Muchos docentes se debaten entre la necesidad de incorporar cambios en la metodología de sus asignaturas y la falta de modelos o instrumentos de diagnóstico para comprobar si los cambios que introducen surten efecto y si se encaminan en la dirección correcta. En este sentido, la validación del JDS adaptado a la docencia universitaria, que presentamos en esta investigación, permite solventar en cierta medida la deficiencia planteada y orientar las acciones de transformación de la metodología docente.

Los profesores que imparten temas relacionados con gestión de recursos humanos, motivación, diseño de puestos de trabajo o JDS, como contenido de sus asignaturas pueden utilizar el cuestionario, que presentamos en esta investigación, como un ejemplo de aplicación de instrumentos profesionales que sea significativo para sus alumnos, analizando el entorno docente que se les propone en diferentes asignaturas.

Por último, cualquier docente que quiera conocer la satisfacción de sus alumnos con su docencia puede disponer de un procedimiento robusto y contrastado que puede complementar o matizar la información que nos llega por las encuestas de alumnado u otras fuentes.

\section{REFERENCIAS}

Anson, C. M. y otros cinco autores; Empowerment to Learn in Engineering: Preparation Foran Urgently-Needed Paradigm Shift, Global Journal of Engineering Education: 7(2), 145-155 (2003).

Armstrong, M. J.; Students As Clients: A Professional Services Model for Business Education, Academy of Management Learning \& Education: 2(4), 371-374 (2003).

Bentler, P.M. Comparative fit indexes in structural models, Psychological Bulletin, 107(2), 238-246 (1990).

Bentler, P.M. y Bonett, D.G. (1980). Significance tests and goodness of fit in the analysis of covariance structures,. Psychological Bulletin, 88, 588-606 (1980).

Bollen, K. A. (ed.).Structural equations with latent variables". John Wiley \& Sons, New York, (1989).

Bollen, K.A. y Long, J.S. Testing structural equation models ". (Eds.) Newbury Park, California: Sage, (1993).

Bolton, M. K.; The Role of Coaching in Student Teams: A "Just-in-Time" Approach to Learning, Journal of Management Education: 23(3), 233-250 (1999).

Boonzaier, b.; B. Ficker y B. Rust; A Review of Research on the Job Characteristics Model and the Attendant Job Diagnostic Survey., South African Journal of Business Management: 32(1), 11-35 (2001).

Brown, M. W y Cudeck, R. "Alternative ways of assessing model fit ". In Bollen, K.A. and Long, J.S. (Eds.) Testing structural equation models, 136-162. N.ewbury Park, California: Sage, (1993).

Cascio, W.; Managing Human Resources: Productivity, QWL, Profits, McGraw-Hill,(1989).

Christoforou, A. P. y otros ocho autores; Improving Engineering Education at Kuwait University Through Continuous Assessment, International Journal of Engineering Education: 19(6), 818-827 (2003). 
Donaldson, L.; Damned by Our Own Theories: Contradictions Between Theories and Management Education, Academy of Management Learning \& Education: 1(1), 96-106 (2002).

Dunham, Randall B.; Ramon Aldag y Arthur P. Brief; Dimensionality of Task Design as Measured by the Job Diagnostic Survey. Academy of Management Journal: 20 (2), 209-23(1977).

Dyson, B. J.; Assessing Small-Scale Interventions in Large-Scale Teaching: A General Methodology and Preliminary Data, Active Learning in Higher Education: 9(3), 265-282 (2008).

Fornaciari, C. J. y K. L. Dean; Experiencing Organizational Work Design: Beyond Hackman and Oldham, Journal of Management Education: 29(4), 631-653 (2005).

Freed, J. E.; Creating a Total Quality Environment (TQE) for Learning, Journal of Management Education: 29(1), 60-81 (2005).

French, N. K. y R. V. Chopra; Teachers As Executives, Theory Into Practice: 45(3), 230-238 (2006).

Fried, Y.; Meta-Analytic Comparison of the Job Diagnostic Survey and Job Characteristics Inventory As Correlates of Work Satisfaction and Performance, Journal of Applied Psychology: 76(5), 690-697 (1991).

Fruchter, R.; Dimensions of Teamwork Education, International Journal of Engineering Education: 17(4-5), 426-430 (2001).

Fuertes Martínez, F.; L. Munduate Jaca y M. A. Fortea Bagán; Análisis y rediseño de puestos (adaptación española del cuestionario Job Diagnostic Survey -JDS-), Universidad Jaime I, Castellón (1996).

González, L.; Estructura Factorial y Propiedades Psicométricas De La Versión Castellana Del "Job Diagnostic Survey" (JDS), Psicológica: 18227-251 (1997).

Griffeth, R. W.; Moderation of the Effects of Job Enrichment by Participation: A Longitudinal Field Experiment, Organizational Behavior and Human DEcision Processes: 35(1), 73-93 (1985).

Griffin, R. W.; Effects of Work Redesign on Employee Perceptions, Attitudes and Behaviors: a Long-Term Investigation, Academy of Management Journal: 34(2), 425-435 (1991).

Gueldenzoph, L. E.; Using Teaching Teams To Encourage Active Learning, Business Communication Quarterly: 70(4), 457-462 (2007).

Hackman, J. R. y Oldham, G. R.; Work redesign, Addison- Wesley,(1980).

Hackman, J. R. y G. R. Oldham; Developrnent of the Job Diaginostic Survey, Journal of Applied Psychology: 60(2), 159-170 (1975).

Hackman, J. R.; J. L. Pearce y J. C. Wolfe; Effects of Changes in Job Characteristics on Work Attitudes and Behaviors: A Naturally Occurring Quasi-Experiment, Organizational Behavior and Human Performance: 21(3), 289-304 (1978).

Hair, J. F.; Anderson, R. E.; Tatham, R. L. y Black, W. C., Multivariate data analysis, . $2^{\text {a }}$ edición, New York, Prentice Hall International, Inc. (1995).

Humphreys, P.; V. Lo; F. Chan y G. Duggan; Developing Transferable Groupwork Skills for Engineering Students, International Journal of Engineering Education: 17(1), 59-66 (2001).

Idaszak, J. R , W. P. Bottom, y F. Drasgow; A Test of the Measurement Equivalence of the Revised Job Diagnostic Survey: Past Problems and Current Solution. Journal of Applied Psychology: 73 (4), 647-656 (1988). 
Jöreskog, K. G. "Testing structural equation models". In K. A. Bollen \& J. S. Long (ed.), Testing structural equation models, pp. 294-316. Newbury Park: Sage, (1993).

Jöreskog, K. G.y Sörbom, D. "Publications. LISREI V. (ed.): Analysis of linear structural relations by the method of maximum likelihood ". Chicago, Illinois: SPSS, (1981).

Jöreskog, K.G. y Sörbom, D. (ed.). Lisrel VI. Analysis of linear structural relationships by Maximum Likelihood, Instrumental Variable, and Least Squares methods ". Moresville, Indiana. Scientific Software. (1986).

Kalliath, T. y M. Laiken; Use of Teams in Management Education, Journal of Management Education: 30(6), 747-750 (2006).

Labrador Piquer, M. J. y Andreu Andrés, M. A.; Metodologías activas, Editorial de la UPV, Valencia (2008).

Lawley, D. N. yd A. E. Maxwell. Factor Analysis as a Statistical Method. Second ed. London: Butterworths. (1971).

Lloret, J. y J. A. Marin-Garcia; Comparing Novel and Stable Lecturers' Point of View When They Use University Students Working Groups in Their Classrooms, WSEAS Transactions on Advances in Engineering Education: 11(5), 699-708 (2008).

Mulaik, Stanley A. y otros cinco autores; Evaluation of goodness-of-fit indices for structural equation models. Psychological Bulletin:105 (3) 430-445 (1989).

Morgeson, F. P. y S. E. Humphrey; The Work Design Questionnaire (WDQ): Developing and Validating a Comprehensive Measure for Assessing Job Design and the Nature of Work, Journal of Applied Psychology: 91(6), 1321-1339 (2006).

O'Neil, D. A. y M. M. Hopkins; The Teacher As Coach Approach: Pedagogical Choices for Management Educators, Journal of Management Education: 26(4), 402-414 (2002).

Rungtusanatham, M. y J. C. Anderson; A Clarification on Conceptual and Methodological Issues Related to the Job Characteristics Model, Journal of Operations Management: 14(4), 357-367 (1996).

Sheppard, K.; P. Dominick y Z. Aronson; Preparing Engineering Students for the New Business Paradigm of International Teamwork and Global Orientation, International Journal of Engineering Education: 20(3), 475-483 (2004).

Smart, K. L. y N. Csapo; Learning by Doing: Engaging Students Through Learner-Centered Activities, Business Communication Quarterly: 70(4), 451-457 (2007).

Swenson, D. X.; You'Ve Just Been Hired-Now What? A Team-Based Business Simulation for an Introduction to Management Course, Journal of Management Education: 25(5), 579-589 (2001).

Taber, T. D.; T. A. Beehr y J. T. Walsh; Relationships Between Job Evaluation Ratings and SelfRatings of Job Characteristics, Organizational Behavior and Human DEcision Processes: 35(1), 27-45 (1985).

Taber, T. D. y E. Taylor; A Reviwev and Evaluation of the Psychometric Propertioes of the Job Diagnostic Survey, Personnel Psychology: 43467-500 (1990).

Tepper, B. J.; S. M. Shafer; J. R. Meredith y R. Marsh; A Clarification on Conceptual and Methodological Issues Related to the Job Characteristics Model: A Reply, Journal of Operations Management: 14(4), 369-372 (1996). 
Terborg, J. R. y G. A. Davis; Evaluation of A New Method for Assessing Change to Planned Job Redesign As Applied to Hackman and Oldham Job Characteristic Model, Organizational Behavior and Human Performance: 29(1), 112-128 (1982).

Van Dijk, L. A. y W. M. G. Jochems; Changing a Traditional Lecturing Approach into an Interactive Approach: Effects of Interrupting the Monologue in Lectures, International Journal of Engineering Education: 18(3), 275-284 (2002).

Watts, F.; A. García-Carbonell y J. Llorens; Introducción a La Evaluación Compartida: Investigación Multidisciplinar, in La Evaluación Compartida: Investigación Multidisciplinar by Watts, F. \& García-Carbonell, A., pp 1-9 Editorial de la UPV, Valencia (2006).

Wenger, M. S. y M. J. Hornyak; Team Teaching for Higher Level Learning: A Framework of Professional Collaboration, Journal of Management Education: 23(3), 311-327 (1999).

Worley, R. B.; Active Learning Strategies, Part 2, Business Communication Quarterly: 71(1), 53-54 (2008). 